\title{
Intensive multidisciplinary treatment strategies and patient resilience to challenge long-term survival in metastatic colorectal cancer: a case report in real life and clinical practice
}

\author{
Gemma Bruera $^{1,2}$, Francesco Pepe ${ }^{3}$, Umberto Malapelle ${ }^{3}$, Mario Di Staso ${ }^{4}$, Antonella Dal Mas ${ }^{5}$, \\ Daniela Di Giacomo ${ }^{1,2}$, Gaia Scerbo ${ }^{6}$, Michela Santilli ${ }^{6}$, Eugenio Ciacco ${ }^{6}$, Maurizio Simmaco ${ }^{7}$, \\ Giancarlo Troncone $^{3}$, Claudio Coco ${ }^{8}$, Felice Giuliante ${ }^{9}$, Enrico Ricevuto ${ }^{1,2}$; on behalf of Oncology \\ Network ASL1 Abruzzo
}

${ }^{1}$ Oncology Territorial Care, S. Salvatore Hospital, Oncology Network ASL1 Abruzzo, University of L'Aquila, L'Aquila, Italy; ${ }^{2}$ Department of Biotechnological and Applied Clinical Sciences, University of L’Aquila, L'Aquila, Italy; ${ }^{3}$ Department of Public Health, University Federico II, Napoli, Italy; ${ }^{4}$ Radiotherapy, S. Salvatore Hospital, Oncology Network ASL1 Abruzzo, L’Aquila, Italy; ${ }^{5}$ Pathology, S. Salvatore Hospital, Oncology Network ASL1 Abruzzo, L’Aquila, Italy; ${ }^{6}$ Pharmacy Unit, S. Salvatore Hospital, Oncology Network ASL1 Abruzzo, L'Aquila, Italy; ${ }^{7}$ Advanced Molecular Diagnostics, S. Andrea Hospital, Rome, Italy; ${ }^{8}$ Unit of General Surgery, Department of General Surgery, Catholic University of Sacred Heart, Rome, Italy; ${ }^{9}$ Unit of Hepatobiliary Surgery, Department of General Surgery, Catholic University of Sacred Heart, Rome, Italy

Correspondence to: Prof. Enrico Ricevuto. U.O.S.D. Oncology Territorial Care, S. Salvatore Hospital, Oncology Network ASL1 Abruzzo, University of L'Aquila, L'Aquila, Italy; Department of Biotechnological and Applied Clinical Sciences, University of L'Aquila, Via Vetoio, L'Aquila, 67100, Italy. Email: enrico.ricevuto@univaq.it.

\begin{abstract}
In fit metastatic colorectal cancer (MCRC), multidisciplinary treatment strategy integrating intensive FIr-B/FOx triplet chemotherapy associated to bevacizumab and secondary metastasectomies significantly improved clinical outcomes up to progression-free survival (PFS) 17 months and overall survival (OS) 44 months. A non-elderly woman affected by rectal cancer, lymph nodes involvement, synchronous unresectable liver metastases, was treated with first-line FIr-B/FOx integrated with two-stage liver resections, short course radiotherapy, anterior rectal resection, with a PFS 9 months and progression-free interval (PFI) 4 months off-treatment. After progression characterized by single liver and lymph node inferior mesenteric axis metastases, FIr-B/FOx was re-introduced, liver and lymph node resections were performed, with a PFS 8 months and PFI 3 months. FIr-B/FOx was further proposed due to bilateral lung, and liver metastases with stable disease, PFS 8 months. Patient experienced a limiting toxicity syndrome multiple sites (LTS-ms) with G3 diarrhea, G2 asthenia, nausea, requiring irinotecan reduction and 5-fluorouracil discontinuation, and subsequent oxaliplatin discontinuation, due to infusional hypersensitivity reaction. Overall, integrated first-line medical and surgical treatment strategies gained PFS 26 months. Further lines II-V of treatment obtained a combined PFS 28 months: modulated aflibercept/irinotecan, PFS 8 months; panitumumab, PFS 8 months, proposed due to KRAS/NRAS/BRAF wild-type and EGFR c.2156 G>C (p.G719A) mutation, achieving biomarkers reduction, lung, liver, lymph nodes partial responses; regorafenib, PFS 8 months; trifluridine-tipiracil, PFS 4 months and induced an LTS-ms, with febrile G4 leucopenia, G3 neutropenia, thrombocytopenia, asthenia, G2 anemia, diarrhea, hypotension. After 2 months of palliative care, patient died, at OS 58 months, gained by intensive medical/surgical treatments coupled with patient's resilience. To date, selection of tailored medical treatments, according to clinical (age, performance and comorbidity status) and molecular (RAS/BRAF and pharmacogenomic analyses) evaluations, careful monitoring of individual toxicity syndromes, potential integration of metastasectomies, and furthermore individual resilience as patient life priority need to challenge MCRC long-term survival.
\end{abstract}

^ ORCID: Gemma Bruera, 0000-0002-8407-2479; Enrico Ricevuto, 0000-0001-5641-3187. 
Keywords: Clinical practice; intensive multidisciplinary treatment strategies in metastatic colorectal cancer; patient resilience; real life; 5 -year survival

Submitted Sep 26, 2020. Accepted for publication Jan 31, 2021.

doi: $10.21037 / \mathrm{atm}-20-6636$

View this article at: http://dx.doi.org/10.21037/atm-20-6636

\section{Introduction}

Effectiveness of first-line medical and surgical treatment strategy of metastatic colorectal cancer (MCRC) is based on activity and efficacy of intensive treatments integrated with secondary resection of metastases, specifically liver metastases, increasing progression-free survival (PFS) up to 17 months (1-6). Decision-making including functional, nutritional, co-morbidity status (Comorbidity Index Rating Scale, CIRS stage) selects patients fit for integrated strategies, or unfit, requiring tailored treatments (7), properly weighing expected safety and efficacy, also according to patient's life priorities.

First-line FIr-B/FOx triplet chemotherapy/bevacizumab reached in fit patients objective response rate (ORR) $82 \%$, PFS 12 months, overall survival (OS) 28 months, $26 \%$ secondary liver resections, $15 \%$ pathologic complete response (CR), significantly improving clinical outcome of liver-limited MCRC, PFS 17 and OS 44 months, compared with multiple metastatic sites $(2,4)$, not significantly affected by KRAS/NRAS/BRAF genotype, even if trendy favourable in triple wild-type (6). In non-elderly $R A S$ wild-type MCRC, first-line FIr-C/FOx-C triplet chemotherapy plus cetuximab was highly active and tolerable at recommended doses, with median PFS 12 months (3). Prognosis after progression to FIr-B/FOx was significantly increased in patients re-challenged with intensive regimens; ORR $80 \%$ correlated with $40 \%$ secondary resections, PFS 13 months, 2 -year OS $80 \%$ (8). Patients unfit for FIr-B/FOx, due to age ( $\geq 75$ years) and/or comorbidities, are prevalent, treated with tailored triplet or doublet chemotherapy, achieving worse clinical outcomes (7).

In patients progressing to oxaliplatin-based first line, aflibercept/FOLFIRI significantly improved clinical outcomes (9). Third-line panitumumab in $R A S$ wildtype (10), regorafenib (11), trifluridine/tipiracil (12) in chemorefractory patients demonstrated significantly increased efficacy.

In clinical practice, careful, continuous monitoring of patient's safety is required to realize integrated multidisciplinary intensive strategies in fit patients. To this aim, we developed the evaluation of limiting toxicity syndromes (LTS) to monitor individual patient toxicity $(2,13-15)$.

We reported a multidisciplinary management in clinical practice of fit, non-elderly, MCRC patient with synchronous, unresectable liver metastases treated with first-line FIr-B/FOx, converted to two-stage hepatectomy and rectal anterior resection, and further lines of treatment, driven by clinical, genetic and pharmacogenomic analyses, who approximately reached 5-year survival, to underline the relevance of multidisciplinary clinical management weighed with individual patient's resilience as life priority to challenge MCRC long-term survival.

We present the following article in accordance with the CARE reporting checklist (available at http://dx.doi. org/10.21037/atm-20-6636).

\section{Case presentation}

Due to right lumbar/abdominal pain, a 59-year-old woman, medical radiologist, with Lynch-like family history (a familial colon, two gastric, a bladder, a testicular cancer), made by herself an ultrasound showing multiple bilateral liver nodules, confirmed at computed tomography (CT) scan, the largest $10-\mathrm{cm}$ diameter, and pathologic rectal enlargement with involved regional lymph nodes. Endoscopic examination confirmed rectal adenocarcinoma, $10 \mathrm{~cm}$ apart from anal verge, NRAS codon 61 c. 183 $\mathrm{A}>\mathrm{T}(\mathrm{Q} 61 \mathrm{H})$ mutation; bone scan negative. CIRS stage was stable without comorbidities. Pharmacogenomic analyses revealed an alteration of irinotecan metabolism (3), consisting of $U G T 1 A 1 * 286 \mathrm{R} / 7 \mathrm{R}$ heterozygote variable number of tandem repeats (VNTR). Due to bilateral liver involvement, locally advanced rectal cancer with nodal involvement, medical oncologist, hepatobiliary surgeon, radiotherapist shared indication to systemic therapy. Patient underwent first-line intensive FIr-B/FOx (2): irinotecan $160 \mathrm{mg} / \mathrm{m}^{2} \mathrm{~d} 1,15$, oxaliplatin $80 \mathrm{mg} / \mathrm{m}^{2} \mathrm{~d} 8,22$, bevacizumab $5 \mathrm{mg} / \mathrm{kg} \mathrm{d} 1,15$, timed-flat- 
Table 1 Timeline of patients' cancer history

\begin{tabular}{|c|c|c|c|c|c|c|}
\hline Timeline & Treatment & No. cycles & $\begin{array}{l}\text { Objective } \\
\text { response }\end{array}$ & PFS (months) & PFI (months) & OS (months) \\
\hline I line & FIr-B/FOx & 4 & PR & 9 & 4 & - \\
\hline Re-introduction & FIr-B/FOx & 8 & SD & 9 & - & - \\
\hline Il line & Flri/aflibercept & 6 & SD & 8 & - & - \\
\hline III line & Panitumumab & 8 & PR & 8 & - & - \\
\hline Exitus & - & - & - & - & - & 58 \\
\hline
\end{tabular}

PFS, progression-free survival; PFI, progression-free interval; OS, overall survival; RT, radiotherapy; PR, partial response; SD, stable disease; PD, progressive disease.

infusion fluorouracil $900 \mathrm{mg} / \mathrm{m}^{2} / \mathrm{d} \mathrm{d} 1-2,8-9,15-16,22-23$, every 28 days, for 3 cycles. Received dose-intensity (rDI) was $>90 \%$ for each drug. Safety profile was characterized by: G2 diarrhea, G1 constipation, nausea, mucositis, asthenia, rhinitis, epistaxis, neuropathy, fever, alopecia. Partial response (PR) of liver metastases was obtained, and of primary rectal cancer, at CT scan, magnetic resonance imaging (MRI), endoscopic evaluation. Multidisciplinary team shared indication to fourth cycle, followed by twostage hepatectomy, short course radiotherapy (RT) and anterior rectal resection with protection ileum stoma. Five weeks after last chemotherapy administration, patient underwent multiple resections at left lobe, and 10 days after, right hepatectomy. Histological examination confirmed multiple metastatic lesions with consistent necrosis (35\% to $>60 \%), 2$ out of 6 metastatic lymph nodes of hepatic artery. Six weeks after, patient underwent short-course RT 5 Gy for 5 days, and 9 weeks after hepatectomy, laparoscopic anterior rectal resection was performed. Histological examination confirmed rectal adenocarcinoma, TRG4 according to Mandard staging, ypT3 ypN2a; 5 out 19 metastatic lymph nodes with capsular invasion. KRAS/NRAS/BRAF wildtype genotype on rectal cancer cells was reported by next generation sequencing (NGS). Patient completed firstline medical treatment, two-stage liver metastasectomies, short-course RT, anterior rectal resection in 8 months; at
PFS 9 months, progression-free interval (PFI) 4 months off-chemotherapy (Table 1), CT scan showed a new third segment liver metastasis $(3 \mathrm{~cm} \times 2.5 \mathrm{~cm})$, and lymph node of inferior mesenteric axis involvement.

Treatment was re-introduced (8) with irinotecan $140 \mathrm{mg} / \mathrm{m}^{2}$ and 5 -fluorouracil $800 \mathrm{mg} / \mathrm{m}^{2} / \mathrm{d}$ doses reduction, due to ileum stoma, 3 cycles. CT scan showed PR, positron emission tomography (PET) negative. At PFS 6 months, 6 weeks after last administration, patient underwent II/ III liver segments, right obturator lymph node resections, and colon canalization restoring. Histological examination confirmed liver and lymph node metastases. At PFS 8 months, PFI 3 months, CT scan showed bilateral lung metastases, a suspected third segment liver nodule $(10 \mathrm{~mm})$. Chemotherapy was resumed with irinotecan $120 \mathrm{mg} / \mathrm{m}^{2}$ and 5 -fluorouracil $750 \mathrm{mg} / \mathrm{m}^{2} / \mathrm{d}$ doses reduction, 3 cycles. Patient experienced LTS-ms (2,13-15): G3 diarrhea, G2 asthenia, G2 nausea, requiring irinotecan reduction $100 \mathrm{mg} / \mathrm{m}^{2}$. Disease was stable, 3 more cycles were planned. From fifth cycle, 5-fluorouracil was discontinued due to LTS single site (LTS-ss) G3 diarrhea; then, oxaliplatin was discontinued, due to severe infusional hypersensitivity reaction. Stable disease was maintained after 8 cycles. At PFS 9 months, CT scan showed progression of third/fourth segments liver metastases; thermoablation $50 \mathrm{~W}$ was performed. Overall, integrated medical/surgical 
first-line treatments obtained PFS 26 months.

Due to described LTS, modulated second-line treatment was proposed: aflibercept $(4 \mathrm{mg} / \mathrm{kg}) \mathrm{d} 1,15$, irinotecan $\left(110 \mathrm{mg} / \mathrm{m}^{2}\right), \mathrm{d} 1,15$, every 28 days. Irinotecan schedule was modified at first cycle, $60 \mathrm{mg} / \mathrm{m}^{2} /$ week. Safety profile was characterized by G2 diarrhea, asthenia, hypertension, G1 rhinitis, epistaxis, mucositis, dysphonia, constipation, nausea. CT scan showed PR of liver, and stable lung metastases, justifying 3 more cycles. Stereotactic liver radiation therapy $54 \mathrm{~Gy} / \mathrm{six}$ fractions was added, determining 4 months later secondary ulcerative hepatic colon flexure lesion, resolved 7 months later. At PFS 8 months, progression of lung and thoracic-abdominal lymph nodes metastases was detected.

Due to KRAS/NRAS/BRAF wild-type genotype by NGS, associated with EGFR c.2156 G>C mutation (p.G719A), third line anti-EGFR panitumumab was proposed, 4 cycles. Consistent biomarkers reduction, $>30 \%$ bilateral lung PR with excavation signs, liver metastases, thoracicabdominal lymph nodes was obtained. Further 4 cycles were administered. At PFS 8 months, CT scan showed increase of thoracic lymph nodes, right shoulder blade metastasis with discontinuation of cortex. During panitumumab, moderately differentiated, ulcerative, infiltrating, cutaneous squamous cell carcinoma of wing of nose was radically resected. Patient underwent RT 800 cGy, cryoablation of bone lesion 13 weeks apart.

Then, fourth-line modulated regorafenib $120 \mathrm{mg} / \mathrm{d}$, 3 weeks on 1 week off was administered, 4 cycles. Safety profile was characterized by: G2 hand-foot syndrome, constipation; G1 diarrhea, hypokalemia, asthenia, anorexia, dysgeusia, mucositis, rhinitis, erythema, fever, rash, paronychia, skin dry, neuropathy. CT scan showed slightly enlarged lung metastases with marked excavation signs, new hepatic and lymph nodes lesions determining right hydro-ureteral distension, requiring placement of ureter stent, stable liver, bone metastases. Three more cycles were administered. At PFS 8 months, CT scan showed increased lung, lymph nodes metastases. Fifth-line trifluridinetipiracil $35 \mathrm{mg} / \mathrm{m}^{2} / \mathrm{bid}, \mathrm{d} 1-5,8-12$ was administered, every 28 days. At first cycle, patient experienced LTSms: febrile G4 leucopenia, G3 neutropenia requiring granulocyte colony stimulating factors and antibiotics, G3 thrombocytopenia, G3 asthenia, G2 anemia, diarrhea, hypotension, associated with G1 nausea, mucositis. Dose was reduced at $25 \mathrm{mg} / \mathrm{m}^{2} /$ bid. After fourth cycle, PFS 4 months, CT scan showed progression of lung, lymph nodes, liver, bone, pelvic metastases. Overall, second-fifth treatment lines obtained PFS 28 months.

No druggable, nor actionable molecular target was detected; ERBB2 and MGMT on liver metastases, MLH1, MSH2, MSH6, PMS2 in primary rectal cancer by immunohistochemistry were normally expressed; microsatellite instability analysis showed stable (MSI-S) genotype.

Then, patient was on best supportive care (BSC); 6 weeks after progression, she was admitted in Palliative Care Unit due to rapidly decreasing clinical conditions and 2 weeks apart she died. De novo metastatic disease OS was 58 months, gained by intensive integration between firstline FIr-B/FOx and secondary resections of liver metastases, short-course RT, primary rectal cancer resection, further second-fifth lines of treatment (Table 1).

All procedures performed in this case report were in accordance with the ethical standards of the institutional research committee and with the Helsinki Declaration (as revised in 2013). Step-by-step, patient was informed about diagnostic, clinical, biologic features, each proposed multidisciplinary treatment strategy. She shared each proposed treatment, signed written informed consent. Written informed consent was obtained for publication of any potentially identifiable images or data included in this article.

\section{Discussion}

The complexity of combined clinical and human features frequently and variably characterizing MCRC patients are summarized in the reported CRC patient who gained OS 58 months, due to the efficacy of subsequent multidisciplinary intensive medical and surgical treatment strategies and, more, due to her resilience to challenge the affecting metastatic disease, as a life priority. Patient was affected by bulky, bilateral liver-limited metastases from locally advanced rectal cancer with lymph nodes metastases. She was fit for multidisciplinary intensive first-line FIr-B/ FOx (2), previously developed from doublet and triplet chemotherapy $(16,17)$, and after a PR, liver metastases were converted to resectability (4), and also resection of primary cancer after short-course RT was performed. Eight months of intensive, integrated medical, surgical, radio-therapeutic approaches converted the bulky into oligometastatic disease, consisting of single liver and inferior mesenteric nodal metastasis, at PFS 9 months. Two further FIr-B/FOx cycles re-introduction were proposed due to PFI 4 and 3 months, respectively, obtained by integrated metastasectomies, gaining overall PFS 26 months and OS 58 months (8). 
In fit patients, first-line FIr-B/FOx reported ORR $82 \%$, secondary liver resections $26 \%$, PFS 12 months, median OS 28 months (2); $>50 \%$ liver metastasectomies, PFS 17 months, OS 44 months in liver-limited $(4,6)$; KRAS exon 2 wild-type liver-limited patients reached significantly prolonged outcome due to secondary surgery, compared to mutant (5). Increased efficacy and liver resections were confirmed in non-elderly $R A S$ wild-type patients treated with intensive $\mathrm{FIr}-\mathrm{C} / \mathrm{FOx}-\mathrm{C}$ triplet chemotherapy associated with cetuximab, reaching PFS 12 months (3). FIr-B/FOx rechallenge, in patients with previous OR, PFS $\geq 10$ months, PFI $\geq 3$ months, no previous limiting toxicities, achieved ORR $80 \%, 40 \%$ secondary resections, PFS 13 months, 2-year OS $80 \%$ (median OS not reached at median followup 31.5 months) (8). Clinical outcome was significantly favourable in re-challenged patients, unfavourable in c. 35 $\mathrm{G}>\mathrm{A}$ KRAS mutant $(8,18,19)$.

In the reported patient, further lines of treatment raised OS 31 months from second line treatment: second line modified schedule/dose aflibercept/irinotecan gaining PR of liver metastases, integrated with stereotactic radiation therapy, PFS 8 months; third line anti-EGFR panitumumab, driven by KRAS/NRAS/BRAF wild-type and EGFR c.2156 G>C mutation (p.G719A), PR of bilateral lung, liver, lymph nodes metastases, PFS 8 months; fourth line regorafenib achieved a stable disease, PFS 8 months; fifth line trifluridine-tipiracil, PFS 4 months before markedly disease progression. In patients resistant or progressing after oxaliplatin-based first-line treatment, aflibercept/ FOLFIRI significantly improved OS 13.5 months, PFS 6.9 months, ORR $19.8 \%$, and $22.3 \%$ of patients reached 30 months OS; in real life, PFS 5.3-6.8 months, OS 12 months, ORR $21.8 \%$ were reported; modified schedules/ doses required in $>50 \%$ of patients did not significantly affect clinical outcomes. Synchronous MCRC patients showed significantly worse PFS 5 months, OS 10 months, while left-sided longer PFS 7 months, OS 12 months. Prevalently reported limiting toxicities were diarrhea, asthenia, mucositis, neutropenia, hypertension (9).

In KRAS/NRAS wild-type, second-line panitumumab/ FOLFIRI reached PFS 6.4 months. In early trial, panitumumab demonstrated efficacy after first and second lines failed (10). Concomitant EGFR mutation, reported in $1.6 \%$ colorectal cancer, even more justified third-line panitumumab treatment, achieving PFS 8 months; EGFR exon 18 c.2156 G>C (p.G719A) is a deleterious, gain-offunction mutation with predictive relevance. Other EGFR extracellular domain mutations detected after treatment justify acquired resistance to anti-EGFR.

In chemorefractory patients, regorafenib reported OS 6.4 months (11), PFS 1.9 months, disease control rate $41 \%$. In Asian population (60\% treated with VEGF- or EGFRtargeted drugs, or both) greater benefit was reached: OS 8.8 months, PFS 3.2 months, disease control rate $51 \%$. In real-life, OS 5.6 months: patients received $78.9 \%$ planned dose, $76 \%$ dose modifications. Hand-foot skin reaction occurrence during first month was associated with significantly better OS 7.7 months, 6-month OS (61\%). Trifluridine-tipiracil significantly increased OS 7.1 months, 1 -year OS $27 \%$, PFS 2.0 months, disease control rate 44\%; patients received $89 \%$ planned dose, $4 \%$ withdrawal (12). Prevalent G3-4 adverse events were neutropenia 38\%, febrile neutropenia $4 \%$, thrombocytopenia $5 \%$, vomiting $2 \%$, diarrhea $3 \%$.

Multidisciplinary strategies characterized by intensive medical treatments require careful toxicity monitoring, proper clinical management, treatment modulations due to moderate/severe toxicities (1). To better evaluate toxicity burden in individual patients, we recently proposed innovative concept of LTS: LTS-ss, characterized by the limiting toxicity (LT), LTS-ms, including LT associated to G2 or other LT (2,13-15). In FIr-B/FOx treated patients we reported LTS 44\%, mainly represented by LTS-ss (2); in young-elderly $46 \%$, mainly including diarrhea (69.2\%), and significantly more LTS-ms compared to LTS-ss (13-15). Patient experienced LTS-ms at FIr-B/FOx re-introductions, G3 diarrhea, associated with G2 asthenia, nausea, requiring irinotecan reduction, 5-fluorouracil discontinuation. Reported LTS may be favored by mutations of genes affecting fluoropyrimidine and/or irinotecan metabolism, mainly including dihydropyrimidine dehydrogenase (DPYD) and UGT1A1 genes, justifying inter-patient safety variability (20); in patients treated with triplet chemotherapy (COI regimen), plus bevacizumab or cetuximab, independent significant association with severe toxicity and treatment modifications was found for DPYD and a trend for UGT1A1 28 mutations $(\mathrm{P}=0.054)(20)$. In patients treated with FIr-C/FOx-C (3), reduced FUDR, UGT1A1 28 , and CYP3A4 single-nucleotide polymorphisms (SNPs) were prevalently detected in patients with LTS and may predict individual LTS occurrence, specifically gastrointestinal; most patients (65\%), specifically developing gastrointestinal LTS (78\%), showed $>1$ pharmacogenomic alteration (1-3). In reported patient, safety profile of further treatment lines was characterized by specific toxicity syndromes; fifth-line trifluridine-tipiracil by LTS-ms. Concomitant occurrence of 
several, different toxicities in individual patient confirmed relevance of evaluating individual toxicity syndromes (15).

Overall, reported patient affected by de novo, bulky metastatic disease gained OS 58 months, PFS 26 months of integrated first line, OS 31 months from second line, by intensive, multidisciplinary treatment strategies, requiring careful, continuous toxicity monitoring, patient's resilience for such a long commitment to complex, continuous therapeutic pathway, including several treatments lines, surgical resections, topical treatments. Careful evaluation of toxicity syndromes represents a clinical parameter measuring how much patient suffers from oncological treatments, indicating individual resilience. Step-by-step, patient was informed about diagnostic, clinical, biologic features of disease and multidisciplinary strategy. Clinical management was shared with patient, weighing each proposed treatment with patient's priorities, regarding options, safety, implications on daily living, modulations/ suspensions, surgery and integration of interventional approaches. In individual MCRC patient fit for intensive treatments, clinical outcome and care also depends upon resilience, careful monitored at each step of disease evolution.

\section{Conclusions}

In clinical practice, selection of MCRC patients eligible for intensive medical treatments to achieve optimal activity and long-term $O S$ by close integration with surgical strategies should evaluate biological ( $R A S / B R A F)$, clinical (age, performance status, CIRS) parameters, integrated with careful monitoring of individual safety using LTS, and patient's resilience as life priority to challenge long-term survival.

\section{Acknowledgments}

We would like to thank "Associazione Humanitas L'Aquila", L'Aquila, Italy (https://www.humanitasaq.org), that supports cancer patient's care for contribution to the article publication fee.

Funding: None.

\section{Footnote}

Reporting Checklist: The authors have completed the CARE reporting checklist. Available at http://dx.doi.org/10.21037/ atm-20-6636
Conflicts of Interest: All authors have completed the ICMJE uniform disclosure form (available at http://dx.doi. org/10.21037/atm-20-6636). The authors have no conflicts of interest to declare.

Ethical Statement: The authors are accountable for all aspects of the work in ensuring that questions related to the accuracy or integrity of any part of the work are appropriately investigated and resolved. All procedures performed in studies involving human participants were in accordance with the ethical standards of the institutional and/or national research committee(s) and with the Helsinki Declaration (as revised in 2013). Written informed consent was obtained from the patient for the publication of any potentially identifiable images or data included in this article.

Open Access Statement: This is an Open Access article distributed in accordance with the Creative Commons Attribution-NonCommercial-NoDerivs 4.0 International License (CC BY-NC-ND 4.0), which permits the noncommercial replication and distribution of the article with the strict proviso that no changes or edits are made and the original work is properly cited (including links to both the formal publication through the relevant DOI and the license). See: https://creativecommons.org/licenses/by-nc-nd/4.0/.

\section{References}

1. Bruera G, Ricevuto E. Intensive chemotherapy of metastatic colorectal cancer: weighing between safety and clinical efficacy: Evaluation of Masi G, Loupakis F, Salvatore L, et al. Bevacizumab with FOLFOXIRI (irinotecan, oxaliplatin, fluorouracil, and folinate) as firstline treatment for metastatic colorectal cancer: a phase 2 trial. Lancet Oncol 2010;11:845-52. Expert Opin Biol Ther 2011;11:821-4.

2. Bruera G, Santomaggio A, Cannita K, et al. "Poker" association of weekly alternating 5-Fluorouracil, Irinotecan, Bevacizumab and Oxaliplatin (FIr-B/FOx) in first-line treatment of metastatic colorectal cancer: a phase II study. BMC Cancer 2010;10:567.

3. Bruera G, Massacese S, Pepe F, et al. Intensive firstline $\mathrm{FIr}-\mathrm{C} / \mathrm{FOx}-\mathrm{C}$ association of triplet chemotherapy plus cetuximab in RAS wild-type metastatic colorectal cancer patients: preliminary phase II data and prediction of individual limiting toxicity syndromes by pharmacogenomic biomarkers. Ther Adv Med Oncol 
2019;11:178835919846421.

4. Bruera G, Cannita K, Giuliante F, et al. Effectiveness of liver metastasectomies in Metastatic Colorectal Cancer (MCRC) patients treated with triplet chemotherapy plus bevacizumab (FIr-B/FOx). Clin Colorectal Cancer 2012;11:119-26.

5. Bruera G, Cannita K, Di Giacomo D, et al. Prognostic value of KRAS genotype in metastatic colorectal cancer (MCRC) patients treated with intensive triplet chemotherapy plus bevacizumab (FIr-B/FOx) according to extension of metastatic disease. BMC Medicine 2012;10:135.

6. Bruera G, Pepe F, Malapelle U, et al. KRAS, NRAS and BRAF mutations detected by next generation sequencing, and differential clinical outcome in metastatic colorectal cancer (MCRC) patients treated with first line FIr-B/ FOx adding bevacizumab (BEV) to triplet chemotherapy. Oncotarget 2018;9:26279-90.

7. Bruera G, Cannita K, Giordano AV, et al. Prognostic relevance of KRAS genotype in metastatic colorectal cancer patients unfit for FIr-B/FOx intensive regimen. Int J Oncol 2014;44:1820-30.

8. Bruera G, Cannita K, Giordano AV, et al. Differential prognosis of metastatic colorectal cancer patients postprogression to first line triplet chemotherapy plus bevacizumab, FIr-B/FOx, according to second line treatment and KRAS genotype. Int J Oncol 2014;44:17-26.

9. Van Cutsem E, Tabernero J, Lakomy R, et al. Addition of Aflibercept to Fluorouracil, Leucovorin, and Irinotecan improves survival in a phase III randomized trial in patients with Metastatic Colorectal Cancer previously treated with an Oxaliplatin-based regimen. J Clin Oncol 2012; 30:3499-506.

10. Amado RG, Wolf M, Peeters M, et al. Wild-type KRAS is required for panitumumab efficacy in patients with metastatic colorectal cancer. J Clin Oncol 2008;26:1626-34.

11. Grothey A, Van Cutsem E, Sobrero A, et al. Regorafenib monotherapy for previously treated metastatic colorectal cancer (CORRECT): an international, multicentre, randomised, placebo-controlled, phase 3 trial. Lancet 2013;381:303-12.

12. Mayer RJ, Van Cutsem E, Falcone A, et al. Randomized Trial of TAS-102 for refractory Metastatic Colorectal Cancer. N Engl J Med 2015;372:1909-19.

13. Bruera G, Cannita K, Giordano AV, et al. Effectiveness and safety of intensive triplet chemotherapy plus bevacizumab, FIr-B/FOx, in young-elderly Metastatic Colorectal Cancer (MCRC) patients. BioMed Res Int 2013;2013:143273.

14. Bruera G, Russo A, Galvano A, et al. Clinical parameters to guide decision-making in elderly metastatic colorectal cancer patients treated with intensive cytotoxic and antiangiogenic therapy. Oncotarget 2017;8:37875-37883.

15. Bruera G, Ricevuto E. Toxicity-syndromes, patient-related indicator of toxicity burden induced by intensive triplet chemotherapy-based regimens in gastrointestinal cancers with metastatic disease. Front Oncol 2020;10:172.

16. Morelli MF, Santomaggio A, Ricevuto E, et al. Triplet schedule of weekly 5 -Fluorouracil and alternating irinotecan or oxaliplatin in Advanced Colorectal Cancer: a dose-finding and phase II study. Oncol Rep 2010;23(6):1635-40.

17. Ficorella C, Bruera G, Cannita K, et al. Triplet Chemotherapy in patients with metastatic colorectal cancer: toward the best way to safely administer a highly active regimen in clinical practice. Clin Colorectal Cancer 2012;11:229-37.

18. Bruera G, Cannita K, Di Giacomo D, et al. Worse prognosis of KRAS c.35 G > A mutant metastatic colorectal cancer (MCRC) patients treated with intensive triplet chemotherapy plus bevacizumab (FIr-B/FOx). BMC Medicine 2013;11:59.

19. Bruera G, Cannita K, Tessitore A, et al. The prevalent KRAS exon 2 c. $35 \mathrm{G}>$ A mutation in metastatic colorectal cancer patients: a biomarker of worse prognosis and potential benefit of bevacizumab-containing intensive regimens? Crit Rev Oncol Hematol 2015;93:190-202.

20. Bruera G, Ricevuto E. Pharmacogenomic assessment of patients with colorectal cancer and potential treatments. Pharmgenomics Pers Med 2020;13:601-17.

Cite this article as: Bruera G, Pepe F, Malapelle U, Di Staso M, Dal Mas A, Di Giacomo D, Scerbo G, Santilli M, Ciacco E, Simmaco M, Troncone G, Coco C, Giuliante F, Ricevuto E; on behalf of Oncology Network ASL1 Abruzzo. Intensive multidisciplinary treatment strategies and patient resilience to challenge long-term survival in metastatic colorectal cancer: a case report in real life and clinical practice. Ann Transl Med 2021;9(12):1027. doi: 10.21037/atm-20-6636 